\title{
Manipulator State Estimation with Low Cost Accelerometers and Gyroscopes
}

\author{
Philip Roan, Nikhil Deshpande, Yizhou Wang, and Benjamin Pitzer
}

\begin{abstract}
Robot manipulator designs are increasingly focused on low cost approaches, especially those envisioned for use in unstructured environments such as households, office spaces and hazardous environments. The cost of angular sensors varies based on the precision offered. For tasks in these environments, millimeter order manipulation errors are unlikely to cause drastic reduction in performance. In this paper, estimates the joint angles of a manipulator using low cost triaxial accelerometers by taking the difference between consecutive acceleration vectors. The accelerometer-based angle is compensated with a uniaxial gyroscope using a complementary filter to give robust measurements. Three compensation strategies are compared: complementary filter, time varying complementary filter, and extended Kalman filter. This sensor setup can also accurately track the joint angle even when the joint axis is parallel to gravity and the accelerometer data does not provide useful information. In order to analyze this strategy, accelerometers and gyroscopes were mounted on one arm of a PR2 robot. The arm was manually moved smoothly through different trajectories in its workspace while the joint angle readings from the on-board optical encoders were compared against the joint angle estimates from the accelerometers and gyroscopes. The low cost angle estimation strategy has a mean error $1.3^{\circ}$ over the three joints estimated, resulting in mean end effector position errors of $6.1 \mathrm{~mm}$ or less. This system provides an effective angular measurement as an alternative to high precision encoders in low cost manipulators and as redundant measurements for safety in other manipulators.

Index Terms-MEMS, accelerometers, gyroscopes, manipulator state estimation, extended Kalman filter, complementary filter
\end{abstract}

\section{INTRODUCTION}

Reducing overall cost is critical to the commercialization of robotic manipulator technologies [1], [2], especially those envisioned for use in unstructured environments such as households, office spaces and hazardous environments [3], [4]. In order for humanoid robots such as the PR2 [2], NAO [5], and others to reach their target market in households and workplaces, the cost of the robot must decrease.

Angular sensors form a critical component of the overall cost of manipulator arm joints [6], which in turn are a significant part of the overall cost of a robot [2]. The cost of angular sensors varies based on the precision offered. Table I shows a cost comparison for the PR2 manipulator arm between traditional optical encoders and MEMS accelerometers and

P. Roan and B. Pitzer are with Robert Bosch LLC. Research and Technology Center, Palo Alto, CA 94304, USA \{philip.roan; benjamin.pitzer\} dus.bosch.com

N. Deshpande is with the Department of Electrical and Computer Engineering, North Carolina State University, Raleigh, NC 27695, USA nadeshpa@ncsu . edu

Y. Wang is with the Department of Mechanical Engineering, UC Berkeley, Berkeley, CA 94720, USA yzhwang@berkeley.edu

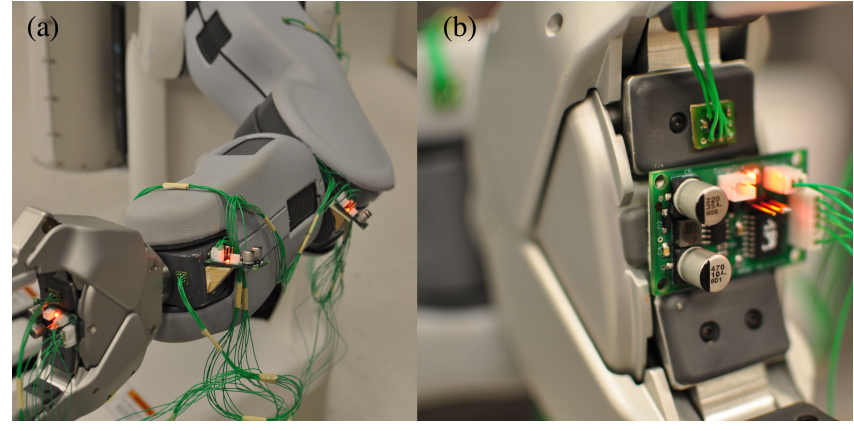

Fig. 1. (a) Photograph of the manipulator arm equipped with accelerometers and gyroscopes. (b) Close-up showing the accelerometer (top) and gyroscope (bottom) for measuring wrist roll.

TABLE I

SENSOR COST COMPARISON

\begin{tabular}{|l|c|c|}
\hline Device & Cost & Total for PR2 Arm \\
\hline \hline Optical Encoder $(1,024$ pulse/rev $)$ & $\$ 40$ & $\$ 280$ \\
\hline Optical Encoder $(10,000$ pulse/rev $)$ & $\$ 110$ & $\$ 770$ \\
\hline MEMS Accelerometer & $\$ 3.11$ & $\$ 22$ \\
\hline MEMS Gyroscope & $\$ 4.77$ & $\$ 33$ \\
\hline
\end{tabular}

gyroscopes [7], [8], [9]. The PR2 arm has 7 degrees of freedom, and one optical encoder can be replaced by one MEMS accelerometer and gyroscope pair.

The use of precision MEMS devices has the potential to offer cost reductions over 5 times over traditional sensors. Although using MEMS accelerometers and gyroscopes may result in lower precision measurements, for tasks in households and workplaces though, millimeter order manipulation errors are unlikely to cause drastic reduction in performance [10].

The cost of accelerometers and gyroscopes has dropped to a point where they have become ubiquitous sensors, as seen by their presence in mobile phones and video game controllers. It is possible to use these sensors to estimate the joint positions of a robotic manipulator [6], [10]. This allows the joint angle sensor to be decoupled from the physical joint axis or actuator axis. In turn, this allows for a truly redundant measurement mechanism for safety as well as for new, lowercost manipulator designs.

Replacing precision optical encoders with accelerometers and gyroscopes may reduce accuracy and repeatability. Gyroscopes are subject to drift as a result of integration of the readings and temperature effects. Accelerometers are unable to capture rotations about an axis parallel to the gravity 
vector. If the manipulator is able to move in this manner, then gyroscopes are required for measuring over the full workspace [10].

This paper presents a strategy to estimate joint angular positions of revolute joints in rigid bodies using low-cost MEMS accelerometers and gyroscopes. Joint angles are estimated using one triaxial accelerometer on each link of a PR2 robot, which is compensated with a uniaxial gyroscope to give robust measurements. The paper includes a comparison of common filtering techniques for combining measurements, such a simple complementary filter [11], [12], [13]; a time varying complementary filter (TVCF) [14]; and an Extended Kalman Filter (EKF) [15]. Two photographs of the manipulator arm equipped with sensors for testing this strategy are shown in Figure 1.

Section II discusses previous work in this field. Section III discusses the theoretical setup, assumptions, and the system model for the proposed strategy. Section IV discusses the tested filtering techniques. Section V provides a physical implementation of the strategy with experimental results.

\section{RELATED WORK}

Early work in utilizing inertial sensors is mostly focused on human motion capture and inertial navigation systems. The low-cost advantages of inertial sensors lend to them being used in human gait and biomechanical analysis. Several such units are utilized in the video-game controller industry [16] as well as in virtual reality and gesture recognition, where the units can be worn by humans on hands and legs [17]. Fong and Chan [18] provide a comprehensive review of the sensing methods and analysis adopted by several researchers in this field. The combination of accelerometers and gyroscopes as inertial measurement units (IMUs) is prevalent in most attitude and orientation tracking systems, for example [19]. Quaternion-based approaches are popular in orientation estimation for applications like aerial vehicles. Here, the device can easily pass through the "gimbal lock" singularity of an Euler Angle solution approach. [20], [21], [22] describe various methods, including integrating the angular rate from the gyroscopes to estimate the headings and using the accelerometer values to stabilize and compensate the gyroscope drift.

In robotic manipulator state estimation, Quigley et al. [10] present an EKF-based method which utilizes the acceleration vector to estimate the joint angle using one accelerometer for each pair of manipulator joints. The kinematics of the manipulator arm are used in the state estimation, and the joint angles are accurately estimated within approximately $1^{\circ}$ of the shaft encoder readings. The authors specifically mention avoiding the singularity condition, when a joint axis is near parallel to the gravity vector, in their experiments. Cheng and Oelmann provide a review and analysis of the various accelerometer-based, gyroscope-compensated rigidbody joint angle estimation techniques [6], [23]. The authors classify the methods into four categories: Common Mode Rejection (CMR), CMR with gyroscope integration, CMR with gyroscope differentiation, and distributed CMR. In the

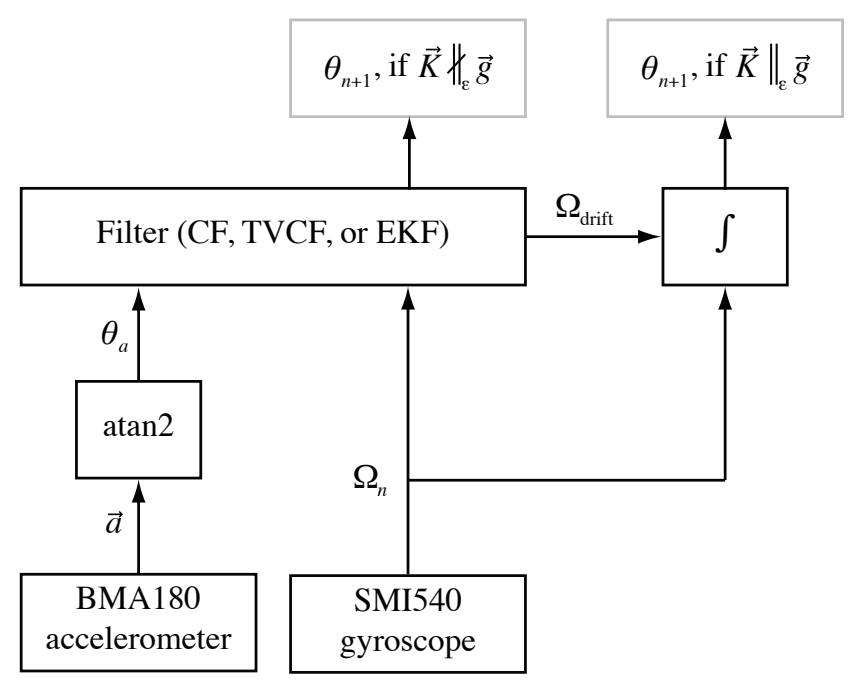

Fig. 2. The proposed method for computing the next joint angle estimate from accelerometer and gyroscope readings at sample $n$. The calculation procedure depends on whether or not the axis of rotation, $\vec{K}$, is parallel within some $\epsilon$ to the gravity vector, $\vec{g}$, or not. The arctangent is used to estimate the joint angle, $\theta_{a}$, from the accelerometer measurements. A filter combines this estimate with the gyroscope measurement, $\Omega_{n}$, to produce a final joint angle estimate for the next time step: $\theta_{n}$.

CMR method, joint angle are calculated from acceleration vectors of two consecutive accelerometers. However, the authors only consider planar manipulator rotations and do not consider non-planar joint rotations such as those likely performed by a manipulator in an unstructured environment.

The above research demonstrates the significant utility of inertial sensors in robotic manipulator joint angle estimation. The combination of accelerometer and gyroscope for 3dimensional motion estimation is an attractive option for reduced cost mechanisms. This paper implements the CMR method in the general non-planar case, and it compares methods of combining accelerometer and gyroscope measurements for joint angle estimates.

\section{ACCELEROMETER BASED ESTIMATION}

The state of a three-dimensional robotic manipulator considered here is the joint angles and velocities. By incorporating a gyroscope on each link, the joint velocities can be directly and accurately measured. Joint acceleration is not considered. Using a priori knowledge of the manipulator kinematics and the corresponding accelerometer mounting, a unified state estimate for the joint angles can be obtained. A critical requirement for the proposed approach is the accelerometer must be able to sense the acceleration due to gravity at all times. Currently, the proposed approach utilizes the gravity component of the acceleration while ignoring the centripetal and centrifugal components. The robot moves slowly to be safe around humans, and the movements are slow enough to justify this assumption. However, any high frequency motions of the manipulator arm may result in unstable readings from the accelerometers, thereby introducing offsets which cannot be compensated. 
Figure 2 provides an overview of how the proposed strategy computes the $(n+1)^{\text {th }}$ estimate of a joint angle, $\theta_{n+1}$, from the accelerometer and gyroscope readings. First, the calculation procedure depends on whether or not the axis of rotation, $\vec{K}$, is parallel within some $\epsilon$ to the gravity vector, $\vec{g}$, or not. If $\vec{K} \nVdash_{\epsilon} \vec{g}$, then the accelerometer input is useful. In this case, an estimate of the joint angle of a given link is fused with gyroscope measurements using one of three filters. [12], [24] present a cogent comparison of the performance of complementary and Kalman filters for orientation estimation problems. The estimated joint angle is used to calculate the gyroscope drift. When $\vec{K} \|_{\epsilon} \vec{g}, \theta_{n}$ is estimated from the the gyroscope measurements and the previously computed gyroscope drift.

\section{A. Rotation Axis not Parallel to Gravity Vector}

When the joint axis is not parallel to the gravity vector, the joint angle is estimated using accelerometer measurements and can be compensated using gyroscope measurements. Each accelerometer measures the gravity vector, and these vectors are the used in sequential pairs as an adaptation of the CMR method in [6] for 3-dimensional angle estimation.

Mounting one accelerometer on every link reduces the calculation of the joint angle between the link pairs to the difference between the gravity vectors measured by consecutive accelerometers. The gravity vector provides an orientation for each accelerometer. X-Y-Z Euler angles are used to relate the accelerometer orientations between consecutive links. A quaternion based solution is not necessary because the joints connecting the manipulator links have only one degree of freedom. The X-Y-Z Euler angle rotation matrix is given in [25], and for the $i^{\text {th }}$ link is given by a rotation $R_{i}$. Thus the measurements of the gravity vector in consecutive frames are related by

$$
\vec{g}_{i}=R_{i} \cdot \vec{g}_{i-1}
$$

The angle of joint $i$ along with any fixed rotational offsets are present in $R_{i}$. Given $\vec{g}_{i-1}$ and $\vec{g}_{i}$ from accelerometer measurements, it is possible to solve for $R_{i}$ and $\theta_{i}$.

The fixed rotational offsets in $R_{i}$ come from the manipulator geometry and the physical placement of the accelerometers on the links. Since they are fixed, The rotation matrix can be written as a product of three rotations:

$$
R_{i}=R_{i^{+}} \cdot R_{i, \theta} \cdot R_{i^{-}}
$$

where $R_{i^{+}}$and $R_{i^{-}}$are fixed rotation matrices determined by the mounting of the accelerometers to the manipulator. Intelligent placement of the accelerometers can simplify the fixed rotations, and a calibration routine can be run to find the remaining values. The variable rotation can be written in any valid form of rotation matrix, but for simplicity let

$$
R_{i, \theta}=\left[\begin{array}{ccc}
\cos \theta_{i} & -\sin \theta_{i} & 0 \\
\sin \theta_{i} & \cos \theta_{i} & 0 \\
0 & 0 & 1
\end{array}\right]
$$

Figure 3 shows a generic joint and two links with accelerometers mounted to the links. Note that the accelerometer axes

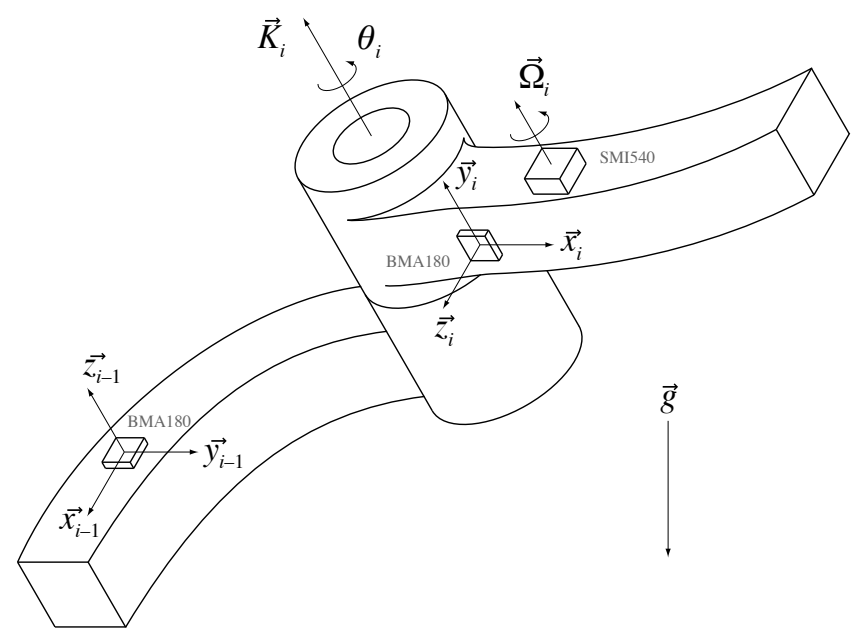

Fig. 3. A generic joint between two links showing how accelerometers and gyroscopes are mounted. The gyroscope axis must be parallel to the axis of joint rotation. The accelerometer axes do not need to be parallel to the axis of rotation, but solving for the angle is simplified if they are.

do not need to be orthogonal to each other or to the joint axis, but the fixed rotation matrix is simplified if they are. This allows the freedom to mount the accelerometer on the link away from the joint itself, as well as in different orientations depending on the mounting considerations.

Equation 3 can be solved using the arctangent. As an example, if $R_{i^{+}}$and $R_{i^{-}}$are identity matrices, then

$$
\left[\begin{array}{l}
x_{i} \\
y_{i} \\
z_{i}
\end{array}\right]=\left[\begin{array}{ccc}
\cos \theta_{i} & -\sin \theta_{i} & 0 \\
\sin \theta_{i} & \cos \theta_{i} & 0 \\
0 & 0 & 1
\end{array}\right]\left[\begin{array}{l}
x_{i-1} \\
y_{i-1} \\
z_{i-1}
\end{array}\right],
$$

Equation 4 provides two transcendental equations in $\theta_{i}$ that can be solved for the joint angle:

$$
\theta_{i}=\operatorname{atan} 2\left(\frac{y_{i} x_{i-1}-x_{i} y_{i-1}}{x_{i} x_{i-1}+y_{i} y_{i-1}}\right) .
$$

Similar equations can be solved for each joint.

\section{B. Rotation Axis Parallel to Gravity Vector (Singularity Condition)}

When a joint axis is parallel to the gravity vector, the accelerometer measurements provide no useful information for determining the joint angle. In this singularity condition, a gyroscope is necessary to estimate the joint angle.

The gyroscope measurements are integrated at each time step to provide the joint angle estimate. There is the potential for large errors to accumulate due to gyroscope drift. Gyroscope drift can be easily calculated when the joint axis is not parallel to the gravity vector by integrating the gyroscope readings and comparing against the joint angle. This is shown in the right half of Figure 2, where the joint angle is most accurately estimated by the output of the filter.

In addition to drift correction, it is expected that a threedimensional manipulator will not spend long periods of time in this singular configuration. 


\section{ACCELEROMETER AND Gyroscope COMPENSATION}

The accelerometer solution presented above is limited to regions of the manipulator workspace where $\vec{K}_{i} \nVdash_{\epsilon} \vec{g}$ for each joint axis. An angular rate gyroscope mounted such that measurement axis is parallel to the axis of rotation of the joint can easily eliminate this restriction.

\section{A. Complementary Filter}

The complementary filter chosen is very simple and efficient to implement. The accelerometer measurements tend to have significant high frequency noise, and the drift in the gyroscope measurements is primarily low frequency. The complementary filter is formed by passing the accelerometer measurements through a low pass filter and the gyroscope measurements through a high pass filter. Using first order IIR filters with the same cutoff frequency yields a complementary filter of the form:

$$
\theta_{i, n}=c\left(\theta_{i, n-1}+\Omega_{i, n} T\right)+(1-c) \theta_{i, a},
$$

where $\theta_{i, n}$ is the $n^{\text {th }}$ estimate of angle $\theta_{i}, \Omega_{i, n}$ is the $n^{\text {th }}$ measurement from the gyroscope, $T$ is the sampling period, $\theta_{i, a}$ is the estimate of $\theta_{i}$ based on acceleration measurements alone, and $c$ is a smoothing constant which determines the cutoff frequency. The time constant for the complementary filter is approximated by

$$
\tau \approx T \frac{c}{1-c} .
$$

\section{B. Time Varying Complementary Filter}

Similar to the complementary filter, the TVCF adjusts the cutoff frequency based on the magnitude of the acceleration vector. The measurement of the gravity vector using accelerometer-based estimation is predominately corrupted by motion accelerations. A time-varying cut-off frequency scheduling can be used to discern the stationary and the moving states, which is proposed by E. Chang-Siu [14]. This is realized by comparing the magnitude of the accelerometer measurement with gravity, or determining the centripetal acceleration based on the gyroscope measurement. Fuzzy logic rules effectively shift the trustworthiness between the gyroscope and the accelerometer. Having a high cutoff frequency indicates that the accelerometer is trusted more, causing the estimate to be more sensitive to accelerometer noises and motion accelerations but attenuate the gyroscope drift, while a low cutoff frequency indicates the gyroscope is the trusted sensor.

\section{Extended Kalman Filter}

Since the relationship between the joint angle and the measured acceleration vectors is non-linear, the EKF is needed over the linear Kalman Filter. In this implementation, a three state EKF is used sequentially; when the EKF for joint $i$ is computed, the a posteriori estimates of the previous $i-1$ joint angles are available. This causes a small loss of accuracy over a monolithic EKF, but it comes with a significant boost in computation speed, as $n 3 \times 3$ matrices are easier to invert than one $3 n \times 3 n$ matrix.
The states are

$$
\left[\begin{array}{c}
\delta \theta_{i} \\
\beta_{i} \\
\ddot{\theta}_{i}
\end{array}\right],
$$

where $\delta \theta_{i}$ is the error in the joint angle estimate from the gyroscope integration, $\beta_{i}$ is the bias in the gyroscope drift, and $\ddot{\theta}_{i}$ is the joint angular acceleration.

The angular rate from the gyroscope is integrated and corrected by $\delta \theta_{i}$. The correction factor is calculated from the measured accelerations and the estimated gyroscope drift to give the final joint angle estimate.

\section{EXPERIMENTS AND RESULTS}

\section{A. Experimental Setup}

The strategy just presented was implemented and tested on a seven degree-of-freedom robot manipulator (PR2, Willow Garage). Five triaxial accelerometers (BMA180, Bosch [7]) and three uniaxial gyroscopes (SMI540, Bosch [26]) mounted on one arm of the robot. Five gyroscopes were not used due to mounting constraints. Linux-based software was written using the open-source Robot Operating System (ROS) platform [27]. The ROS framework provides logging, playback, and visualization tools.

The BMA180 accelerometers provide 14-bit accuracy, adjustable measurement ranges, an SPI interface, and programmable digital filters. The SMI540 gyroscopes provide 16-bit accuracy, low noise, an SPI interface, and a programmable digital filter. Drivers for both sensors are freely available in ROS.

Figure 1 shows two photographs of the sensors mounted on the robot arm. The sensors are mounted such that three angles could be estimated: Elbow Flex, Wrist Flex, and Wrist Roll. Neither sensor type needs to be mounted coincident with the joint axis, but the gyroscope axis is mounted parallel to the joint axis.

\section{B. Numerical Results}

Before the joint angles can be estimated, a calibration step is warranted owing to the misalignments internal to the sensors as well as their mounting on the manipulator arm and the manufacturing tolerances in the manipulator structure. Since the PR2 arm is equipped with high-precision optical encoders in each joint, a simple calibration step is run to estimate the initial offsets and fixed rotations. The PR2 arm is held stationary with the sensors mounted. The angle estimation algorithm is run for 10 seconds at $100 \mathrm{~Hz}$, allowing for the generation of a mean offset for each of the joint angles by comparing the estimated angles with the actual angles from the encoders. This accounts for the misalignments of the kinematic frames of the joints with respect to that of the accelerometers and gyroscopes.

During experimentation, parameters were empirically adjusted to provide the best results including the threshold for when the joint axis is parallel to the gravity vector, the time constant for the complementary filters, and the noise covariances for the EKF. The transition threshold for the $i^{t h}$ 


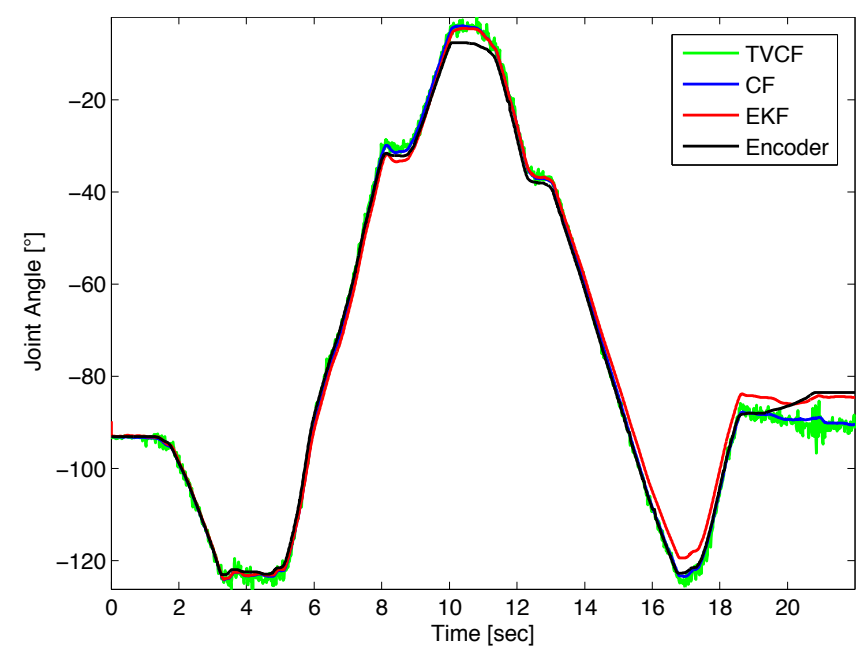

Fig. 4. Angle of the Elbow Flex joint with estimated angles.

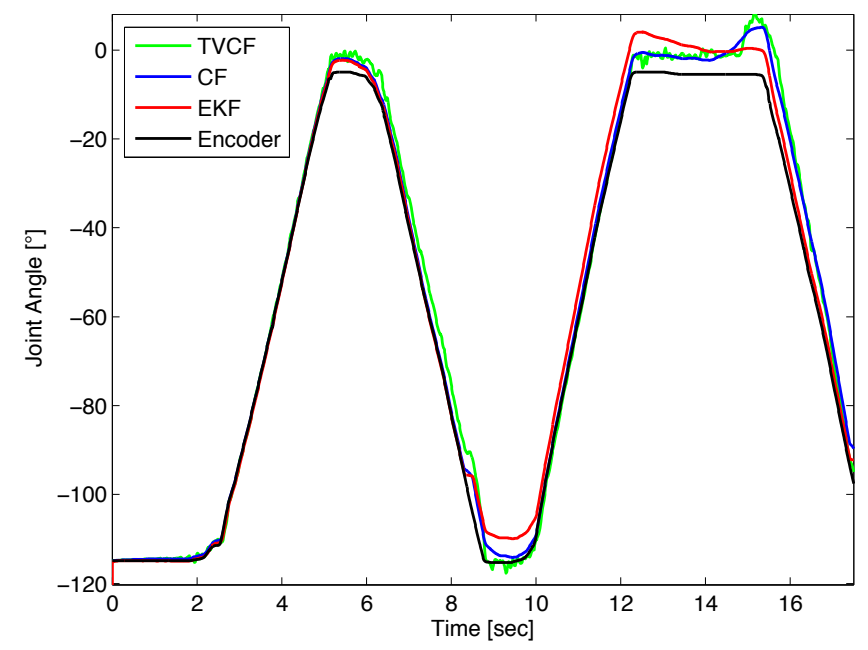

Fig. 5. Angle of the Wrist Flex joint with estimated angles.

joint axis to be vertical, depends on the absolute value of $a_{z}$ in $\vec{a}_{i}$ because the accelerometer is mounted so this axis is parallel to the joint axis. Based on 30 different trials for the angle estimation transition, the threshold is set as $0.85 \mathrm{~g}$. The time constant for the complementary filter is set to 2 seconds.

The arm was manually moved smoothly through different trajectories in its workspace and the four joint angles were recorded over 120 seconds at a rate of $100 \mathrm{~Hz}$. The joint angle readings from the on-board optical encoders were compared against the joint angle estimates from the filters using the accelerometers and gyroscopes. Figures 4, 5, and 6 and show the favorable tracking performance. Table II shows the mean error and standard deviation in the estimated angles for all three joints. The deviations increase for links further from the base, and is likely caused by larger magnitude vibrations of the manipulator at those links.

The Wrist Roll joint shows a larger error in estimation than the Elbow Flex and Wrist Roll joints. This appears to be due to the steps in the encoder signal. Since the joints are moved manually and smoothly, it seems likely that the encoder

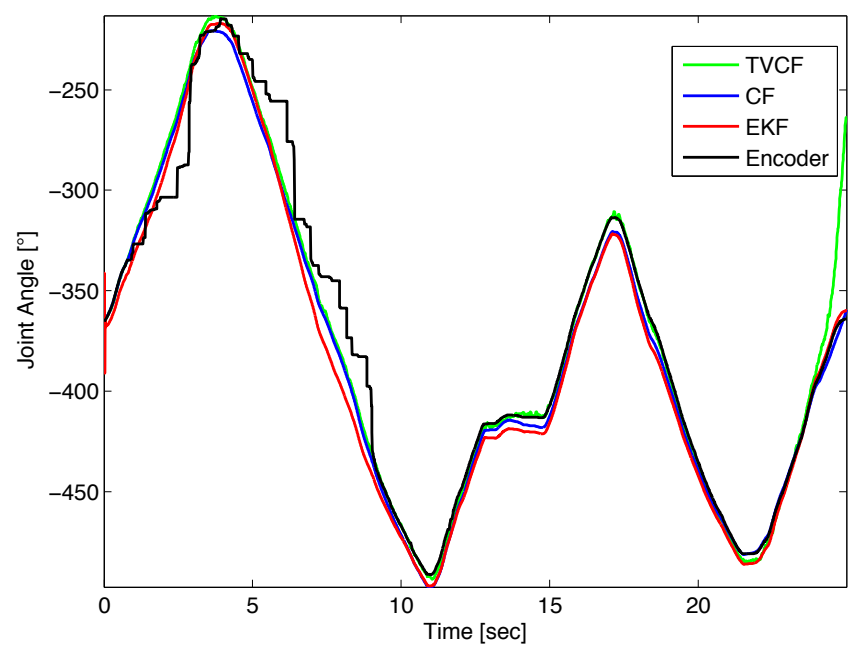

Fig. 6. Angle of the Wrist Roll joint with estimated angles. The steps in the encoder signal give rise to the large errors for this joint. In this case the encoder may be malfunctioning.

is malfunctioning. The Elbow Flex exhibits the smallest standard deviation for all filters. This is expected as there is the the distal sensors experience larger accelerations when the arm is moved. The current filtering strategy assumes that the arm motion is quasistatic where the only accelerations felt by the sensors is from gravity.

\section{Discussion AND Conclusions}

The main advantages in utilizing MEMS accelerometers and gyroscopes for manipulator state estimation are a reduction in costs, a reduction in sensor size, and decoupling angle estimation from the physical joint-actuator axes. The reduction in size is clear from form-factor comparisons of MEMS devices with other regular-sized mechanical components, especially the high-precision encoders used in robot manipulator arms. The reduced size, along with the decoupling from the joint axis, affords more flexibility in sensor placement along the manipulator link. This placement flexibility plays an important role in the development of

TABLE II

JoInt Angle Estimation ERROR (DEGREES)

\begin{tabular}{|l|l|c|c|}
\hline Joint Name & Filter & Avg. Error [ ${ }^{\circ}$ ] & Std. Dev. \\
\hline \hline Elbow Flex & CF & 0.18 & 1.24 \\
\hline Elbow Flex & TVCF & 0.12 & 1.64 \\
\hline Elbow Flex & EKF & 0.54 & 1.18 \\
\hline Wrist Flex & CF & 0.99 & 2.97 \\
\hline Wrist Flex & TVCF & 0.49 & 3.36 \\
\hline Wrist Flex & EKF & 0.82 & 4.76 \\
\hline Wrist Roll & CF & 6.07 & 7.90 \\
\hline Wrist Roll & TVCF & 3.73 & 7.43 \\
\hline Wrist Roll & EKF & 2.46 & 9.59 \\
\hline
\end{tabular}


new, smaller foot-print manipulators that may have space restrictions preventing the use of regular-sized encoders [5].

The main disadvantages of MEMS sensors are a reduction in accuracy and increased measurement noise. The accuracy measured in these experiments is enough to enable a visual feedback loop to make additional corrections. Since most mobile manipulators like the PR2 also include cameras, the ability to use a visual feedback loop is a valid assumption. The visual feedback loop can also compensate for any thermal drift in the MEMS sensors. Additionally, in many tasks millimeter level accuracy is not needed.

By comparing between two sequential links, joint angle errors do not compound. The mean errors in the Elbow Flex produce end effector position errors of $4.5 \mathrm{~mm}, 6.1 \mathrm{~mm}$, and $3.2 \mathrm{~mm}$ for the CF, TVCF, and EKF, respectively. The sensors are small and require a single data bus, reducing the wiring along the arm. The accuracy with which they can track the manipulator state allows them to provide an effective redundant measurement for safety or replace the current measurement system in a low-cost manipulator.

The accelerometer and gyroscope based estimation tracks the manipulator motion quite well. As noted earlier, the accelerometer measurements are noisy, especially for the distal joints of the arm, even with a low sensor sampling rate of $100 \mathrm{~Hz}$. The sampling rate would have to be much higher, close to $1000 \mathrm{~Hz}$, for active feedback-controlled motions of the arm. The sensors are capable of measuring at this frequency, however $100 \mathrm{~Hz}$ was chosen for proof-of-concept purposes, considering that only passive movements of the manipulator were being analyzed.

With the setup described here, only passive movements of the manipulator are analyzed. In the future, experiments will be conducted where the manipulator motion is actively controlled using the estimated joint angles in place of the optical encoders. Improved filtering and noise canceling strategies to produce smooth arm motion without pronounced vibrations. In addition, the existing algorithms assume slow motions, or turn down the trustworthiness of the accelerometer when detecting large motions. Because of the coupled kinematic relations, motion accelerations can actually be utilized in order to obtain a better joint angle estimate by using an extended Kalman filter where the exact kinematics are modeled. These experiments will provide additional information on the reliability and robustness of this type of manipulator state estimation.

\section{REFERENCES}

[1] (2008, February) Major Personal Robotics Components Will Represent a $\$ 12$ Billion Market by 2015. ABI Research. Accessed on 5 Mar. 2012. [Online]. Available: http://www.abiresearch.com/press/1050-Major+Personal+Robotics+ Components+Will+Represent+a+\$12+Billion+Market+by+2015

[2] E. Ackerman. (2011, August) Willow Garage Introduces PR2 SE, Half the Arms at Half the Price. Accessed on 12 Sept. 2011. [Online]. Available: http://spectrum.ieee.org/automaton/robotics/robotics-software/ willow-garage-pr2-se-now-available-half-the-arms-at-half-the-price

[3] M. P. Deisenroth, C. E. Rasmussen, and D. Fox, "Learning to Control a Low-Cost Manipulator using Data-Efficient Reinforcement Learning," in Proceedings of Robotics: Science \& Systems, (RSS), 2011.
[4] J. Wang, et al., "Control Strategy for a Low Cost Manipulator to Transport and Align IC Mask-Plates," IEEE Transactions on Control Systems Technology, vol. 17, no. 5, pp. 1018 -1027, Sept. 2009.

[5] T. Niemüller, et al., "Providing Ground-truth Data for the Nao Robot Platform," in RoboCup 2010, J. Ruiz-del Solar, E. Chown, and P. G. Plöger, Eds. Berlin, Heidelberg: Springer-Verlag, 2011, pp. 133-144. [Online]. Available: http://dl.acm.org/citation.cfm?id= 1983806.1983819

[6] P. Cheng and B. Oelmann, "Joint-Angle Measurement Using Accelerometers and Gyroscopes - A Survey," IEEE Transactions on Instrumentation and Measurement, vol. 59, no. 2, pp. 404 -414, Feb. 2010.

[7] "Bosch BMA180 Digital, Triaxial Acceleration Sensor," Accessed on 10 Mar. 2012. [Online]. Available: http://search.digikey.com/us/en/ products/BMA180/828-1019-2-ND/2416311

[8] "ST Dual Axis Gyroscope: LPY410ALTR," Accessed on 10 Mar. 2012. [Online]. Available: http://search.digikey.com/us/en/products/ LPY410ALTR/497-10860-2-ND/2214497

[9] (2012, March) Incremental Quadrature Optical Encoders - 1024 to 10000 PPR. Digi-Key Corporation. Accessed on 5 Mar. 2012. [Online]. Available: www.digikey.com

[10] M. Quigley, et al., "Low-cost Accelerometers for Robotic Manipulator Perception," in Proc. IEEE/RSJ Intl. Conf. on Intelligent Robots and Systems, (IROS 2010), Oct. 2010, pp. $6168-6174$.

[11] R. G. Brown, "Integrated navigation systems and kalman filtering: A perspective," Navigation, vol. 19, no. 4, pp. 355-362, Winter 19721973.

[12] W. T. Higgins, "A comparison of complementary and kalman filtering," Aerospace and Electronic Systems, IEEE Transactions on, no. 3, pp. 321-325, 1975.

[13] S. Colton, "The Balance Filter," Massachusetts Institute of Technology, Tech. Rep., 2007.

[14] E. Chang-Siu, M. Tomizuka, and K. Kong, "Time-Varying Complementary Filtering for Attitude Estimation," in Intelligent Robots and Systems (IROS), 2011 IEEE/RSJ International Conference on. IEEE, Sept. 2011, pp. 2474-2480.

[15] G. Welch and G. Bishop, "An Introduction to the Kalman Filter," Department of Computer Science, University of North Carolina at Chapel Hill, Tech. Rep. TR 95-041, Jul. 2006.

[16] T. Shiratori and J. K. Hodgins, "Accelerometer-based User Interfaces for the Control of a Physically Simulated Character," ACM Transactions on Graphics, (SIGGRAPH), vol. 27, no. 5, pp. 123:1-123:9, Dec. 2008.

[17] J. Lee and I. Ha, "Real-Time Motion Capture for a Human Body using Accelerometers," Robotica, vol. 19, no. 6, pp. 601-610, 2001.

[18] D. T.-P. Fong and Y.-Y. Chan, "The Use of Wearable Inertial Motion Sensors in Human Lower Limb Biomechanics Studies: A Systematic Review," Sensors, vol. 10, no. 12, pp. 11 556-11 565, Dec. 2010.

[19] K. J. Walchko and P. A. C. Mason, "Inertial Navigation," in Florida Conference on Recent Advances in Robotics, 2002.

[20] E. Kraft, "A Quaternion-based Unscented Kalman Filter for Orientation Tracking," in Proc. $6^{\text {th }}$ Intl. Conf. of Information Fusion, vol. 1, 2003, pp. 47 - 54.

[21] A. Sabatini, "Quaternion based Attitude Estimation Algorithm applied to Signals from Body-mounted Gyroscopes," Electronics Letters, vol. 40, no. 10, pp. 584 - 586, May 2004.

[22] J. Favre, et al., "Quaternion-based Fusion of Gyroscopes and Accelerometers to Improve 3D Angle Measurement," Electronics Letters, vol. 42, no. 11, pp. 612 - 614, May 2006.

[23] F. Ghassemi, et al., "An Accelerometer-based Joint Angle Sensor for Heavy-duty Manipulators," in Proc. IEEE Intl. Conf. on Robotics and Automation, (ICRA '02), vol. 2, 2002, pp. 1771 - 1776.

[24] A. D. Young, "Comparison of Orientation Filter Algorithms for Realtime Wireless Inertial Posture Tracking," in $6^{\text {th }}$ Intl. Workshop on Wearable and Implantable Body Sensor Networks, (BSN 2009), Jun. 2009, pp. $59-64$.

[25] J. J. Craig, Introduction to Robotics: Mechanics and Control, $3^{\text {rd }}$ ed. Upper Saddle River, New Jersey, USA: (c) Pearson Prentice Hall, 2005.

[26] "Bosch SMI540 Angular Rate Sensor," Sept. 2009, Accessed on 12 Sept. 2011. [Online]. Available: http://www.bosch-presse.de/ presseforum/details.htm?txtID $=4101 \&$ locale $=\mathrm{en}$

[27] M. Quigley, et al., "ROS: an open-source Robot Operating System," in Open-Source Software Workshop of the IEEE Intl. Conf. on Robotics and Automation, (ICRA '09), 2009. 